\title{
BMJ Quality Knowledge is Power. A quality improvement project to increase patient understanding of their hospital stay
}

\author{
Eleanor Nicholson Thomas, Lloyd Edwards, Paul McArdle
}

To cite: Nicholson Thomas E, Edwards L, McArdle P. Knowledge is Power. A quality improvement project to increase patient understanding of their hospital stay. BMJ Quality Improvement Reports 2017;6:u207103.w3042. doi:10.1136/bmjquality. u207103.w3042

Received 2 August 2016 Revised 9 September 2016

CrossMark

Plymouth Hospitals NHS Trust

Correspondence to Eleanor Nicholson Thomas enthomas89@gmail.com

\section{ABSTRACT}

Patients frequently leave hospital uninformed about the details of their hospital stay with studies showing that only $59.9 \%$ of patients are able to accurately state their diagnosis and ongoing management after discharge. ${ }^{12}$ This places patients at a higher risk of complications. Educating patients by providing them with accurate and understandable information enables them to take greater control, potentially reducing readmission rates, and unplanned visits to secondary services whilst providing safer care and improving patient satisfaction. $^{3} 4$

We wished to investigate whether through a simple intervention, we could improve the understanding and retention of key pieces of clinical information in those patients recently admitted to hospital.

A leaflet was designed to trigger patients to ask questions about key aspects of their stay. This was then given to inpatients who were interviewed two weeks later using telephone follow up to assess their understanding of their hospital admission. Patients were asked about their diagnosis, new medications, likely complications, follow up arrangements and recommended points of contact in case of difficulty. Sequential modifications were made using PDSA cycles to maximise the impact and benefit of the process.

Baseline data revealed that only $77 \%$ of patients could describe their diagnosis and only $27 \%$ of patients knew details about their new medications. After the leaflet intervention these figures improved to $100 \%$ and $71 \%$ respectively.

Too often patients are unaware about what happens to them whilst in hospital and are discharged unsafely and dissatisfied as a result. A simple intervention such as a leaflet prompting patients to ask questions and take responsibility for their health can make a difference in potentially increasing patient understanding and thereby reducing risk.

\section{PROBLEM}

From our own experiences on the wards and through clerking patients we found that a significant proportion of patients had limited knowledge and understanding of their medical history. This is compounded by a lack of awareness that clinical staff have of the paucity of important information that patients are given and successfully retain. ${ }^{4}$ Further we tolerate that patients are unable to answer important questions such as why they were previously in hospital or why they are on certain medications.

Poor knowledge places patients at risk of complications that might otherwise easily be managed or dealt with by appropriate early interventions. Patients without such knowledge may not know who to contact and may not know whether what they are experiencing is appropriate or untoward. This may result in unplanned visits to primary or secondary care and in some cases in delayed presentations. $^{1}$

The systems in our hospital wards are frequently poorly organised to ensure patients have the best information regarding their care. The busy environment is not the ideal place for learning, with ward rounds in some cases only lasting for a couple of minutes for each patient. Ward rounds are designed for the convenience of the medical staff rather than for patients and assumptions are made that patients understand the medical jargon and systems in the hospital. Patients are rarely encouraged to ask systematic questions about their care and there is often little time for patients to check their understanding. There is an understandable tension to be overcome in both making sure patients are seen and that their care is coordinated.

For this reason a simple intervention that encourages patient ownership and responsibility for their condition through active personal education could make sure patients are able to get the information they require.

The project team consisted of two FY2 doctors and a consultant mentor. Our project was created from our own experiences on the wards during our first year of foundation training. We worked in a busy tertiary centre in Devon with approximately 
1000 beds providing hospital services to 450,000 people in Plymouth, East Cornwall and South Devon. Our project was trialled on inpatients in the two general surgical wards of the hospital which together had 60 beds in total.

\section{BACKGROUND}

Many studies have been done to determine the knowledge that patients have about their inpatient stay, their diagnoses and the medications that they have been started on.

One study revealed that although the vast majority of patients say that they understand the reason why they were in hospital, only $59.6 \%$ of patients were able to accurately describe their diagnosis and future management. ${ }^{2}$ The remaining $40 \%$ of patients do not remain ignorant by choice. A study into the diagnosis of cancer patients revealed that $96 \%$ of patients wanted to know their diagnosis, and more than $90 \%$ wanted to know about their prognosis, the treatment options and the possible side effects. ${ }^{3}$

It has been shown that there are differing perceptions between physicians and patients regarding patient understanding of their management plan. Physicians believed that $95 \%$ of patients understood when to restart normal activities whereas only $57 \%$ of patients agreed. This disparity needs to be addressed in order to improve communication about post-discharge management. ${ }^{4}$

'Self management' is the patient developing an understanding of how their condition affects their lives and how to cope with their symptoms. Self management education for patients with COPD has been shown to reduce the risk of hospital admission by about $36 \%$ compared with standard care. ${ }^{5}$

Interventions to improve patient knowledge have been directly linked to an increase in patient satisfaction with their care. ${ }^{4}$ Evidence has shown that patients who are more satisfied with their care are less likely to be readmitted or seek medical attention from secondary services after discharge. ${ }^{16}$

$8 \%$ of all patient admissions to hospital are readmissions within 30 days. Under the non-payment scheme for acute readmissions, NHS hospitals suffer a loss of 760 million per year as a result of these readmissions. ${ }^{7}$ Any intervention to help reduce this burden could save costs in an already financially unstable system.

\section{BASELINE MEASUREMENT}

To assess the problem, a baseline measurement of patient understanding was needed. The phone numbers of patients discharged from two medical wards over two consecutive weeks were collected from the ward clerk discharge book. The patient was then phoned two weeks later and asked for their consent to participate in our study.
Patients were asked to give 'yes' or 'no' answers to six key questions about their hospital stay and the answer given was documented in an excel spreadsheet. The questions (and percentage of 'yes' answers) included;

Do you know your diagnosis? $(77 \%)$;

Do you know the side effects of the medication you have been started on (if any)? (27\%);

Do you know about possible ongoing health issues that might occur at home? (27\%);

Have you been given details of who to contact if there was a problem? $(43 \%)$;

Have you had any complications at home? (30\%);

Have you had any unplanned contact with your GP after discharge? (43\%)

Of the 60 patients who were contacted, 30 answered their phone and consented to the study.

A positive response to a question means answering 'yes' to "do you know your diagnosis?" or answering 'no' to "have you had any unplanned contact with your GP". The number of positive responses was then divided by the total number of questions and plotted on a run chart. We re-assessed our questions and decided to add an important seventh question asking the patient about the next steps in their care. This question would be asked for the remainder of the study (See supplementary - Background data chart).

\section{DESIGN}

The 'SMART' (specific, measurable, achievable, realistic, timely) aim for this project was to increase the percentage of patients who understand six key pieces of information about their hospital stay from $47 \%$ to $90 \%$ in a 12 month period.

Information leaflets are occasionally available for certain conditions but are not routinely given to patients on discharge in our hospital, and if they are given out, are not patient specific. In addition to this, condition specific leaflets are time consuming and costly to create. We devised a simple leaflet which asked the patient questions about their condition and follow-up aiming to prompt patients to ask for answers from healthcare staff if they were unsure. This was a simple and inexpensive intervention designed to be patient-centred, easily distributable on the wards and with the potential for implementation on other wards and eventually hospital wide.

The details of the patients who were given the leaflets were recorded and a phone call was made two weeks later asking the patient the same questions. Following this first intervention, patient and colleague reaction was assessed, resulting in mostly enthusiastic and positive feedback as well as constructive ideas to improve the leaflet.

\section{STRATEGY}

Several PDSA (Plan,Do,Study,Act) cycles were carried out to ensure successful achievement of our SMART aims. 
For our first PDSA cycle we asked the ward clerk to distribute the question leaflet with other routine discharge paperwork given to the patient on the day of discharge. This was to be given to all patients discharged during the week in May 2014. The leaflet was given out to 23 patients and data was collected from 13 patients who answered their phones. Feedback from the patients highlighted that the paper was too small to be noticed and it got lost within the other discharge documents meaning many patients never saw it. We realised we that the leaflet needed to be larger and distributed at least one day prior to discharge to allow enough time for the patient to ask questions. We considered the importance of patient satisfaction and how this might be improved with education and therefore decided to ask a seventh question for the remaining PDSA cycles 'were you satisfied with your care?'. This PDSA did not significantly improve patient understanding but we learned some valuable lessons for our next cycle.

For the second PDSA cycle we built on patient suggestions and increased the size of the leaflet to A4. It was printed in colour to make it stand out and the questions were re-worded to make them easier to understand. The leaflets were given out by ourselves over the course of a week to those patients with planned discharge for the following day. This included 21 patients of which 11 were contactable via a phone call 2 weeks later. We saw an improvement in knowledge and received excellent feedback from patients, however there was still some misunderstanding of the purpose of the leaflet since a few patients thought it was a quiz that needed to be given back.

In the third PDSA cycle we came up with the final design for the leaflet (Figure 1) which included a short sentence in red at the top of the leaflet to direct the patients' attention and inform them that the paper is for their own use. Additionally lines were added underneath to encourage patients to write notes for themselves. The leaflets were given out by the ward clerk and ourselves over the course of a week to try and improve distribution numbers. The leaflets were given to 27 patients of which 13 were contactable. The next step was to find a method of distribution, which did not rely on ourselves, in order to make the project sustainable on the wards. We planned to do this using those healthcare staff already carrying out their normal tasks on the ward.

The week prior to the fourth PDSA cycle, our project was presented to the ward doctors who were asked to give out the leaflets themselves on the day before discharge. Unfortunately only two leaflets were given out during the week as the doctors found themselves too busy to add another job to their workload. Following this the ward clerk and ward manager were asked to distribute the leaflets instead which gave us a total of only 8 patients for this cycle and 5 of which provided us with answers. We reassessed the distribution method and decided that the most sustainable way was for the nurses to give out the leaflets however in such a busy
The following is for your own information; you are welcome to take this home with you for future reference.

\section{Please read this very carefully} Plymouth Hospitals WHS

Before you leave hospital, make sure you have the answers to these questions:

1) What is my diagnosis?

2) What medications have I been started on and why?

3) What problems could occur at home?

4) Who should I contact if I have any problems at home?

5) What is the next step in my care? Who will I see and when?

If you are unsure please ask a doctor or nurse from the team

Space for your notes:

environment it was low on their priority list. We decided the best way to address this was to have a proper discussion with the nurses to highlight the value of this study on patient care and create enthusiasm amongst staff.

For PDSA five, a meeting with the ward staff was held to discuss the most effective but least time consuming way of distributing the leaflet. The meeting included the clinical director, the nurse manager, ward clerk, three registered nurses and ourselves. It was decided that the nurses would give the patient the leaflet upon arrival to the ward when other admission paperwork was being done. This seemed to be the easiest way and still allowed enough time for patients to ask questions. The feedback from patients was very positive, but still only 13 patients received a leaflet with 6 providing us with data. Our limiting factor to making a sustainable project remained the number of patients receiving the leaflet and so for our sixth PDSA we focused on distribution of the leaflet. 
In our sixth PDSA we took a different approach to address the issue of distribution on the ward. We used the surgical assessment unit (SAU) because of the high turnover of patients. Over a 1 week period, we recorded how many patients were given leaflets by the nurses on arrival to the SAU and then how many of these patients retained or remembered the leaflet when they were moved to the ward. This required the SAU nurse to document in a table which patients were given the leaflet and then we located that patient on the inpatient surgical ward and asked them whether they had retained the leaflet.

\section{RESULTS}

All patients who were given leaflets were telephoned two weeks after discharge and the same questions used in the baseline data collection were asked. After the first PDSA cycle a question was added to assess whether they were satisfied with the knowledge that they had. After each PDSA cycle the knowledge that patients had retained was analysed. An improvement was seen if the percentage of positive responses per patient increased compared with the previous PDSA cycle. These results were plotted onto a run chart (Figure 2).

As the PDSA cycles progressed the mean number of positive responses showed an upward trend from $70 \%$ after PDSA 1 to $91 \%$ after PDSA 5. The median positive responses showed a very similar improvement from $67 \%$ after PDSA 1 to 93\% after PDSA 5. PDSA 6 revealed that $47 \%$ of patients had been given the leaflets on admission, but $18 \%$ had lost it since. This rate of distribution was higher than expected given the decrease in the sample size in the previous PDSA cycles.

There was some expected variation in the results in each PDSA cycle, with the standard deviation in PDSA 1 at $27 \%$, and PDSA 5 at $11 \%$. This is likely due to the variety of patients who were included in the study, with varying ages, medical problems and likely educational backgrounds.

Our baseline results are validated as they are very similar to our PDSA 1 results. Most of the patients did not see the leaflet in PDSA 1 therefore most patients had no intervention. Our results in PDSA 4 and 5 showed a much improved positive response rate of 95 and $93 \%$ in comparison to $47 \%$ at the baseline.

\section{LESSONS AND LIMITATIONS}

Many valuable lessons have been learned during the process of carrying out the project. The appearance of the patient leaflet was a key factor in ensuring the project worked. Initially the format was of a small black and white question card which could be easily lost within the other discharge information given to the patient. In order to be noticed and read, the leaflet needed to be larger with colour to stand out and grab the reader's attention.

\section{A Run Chart Showing the Positive Questionnaire Responses Throughout the PDSA}

\section{Cycles}

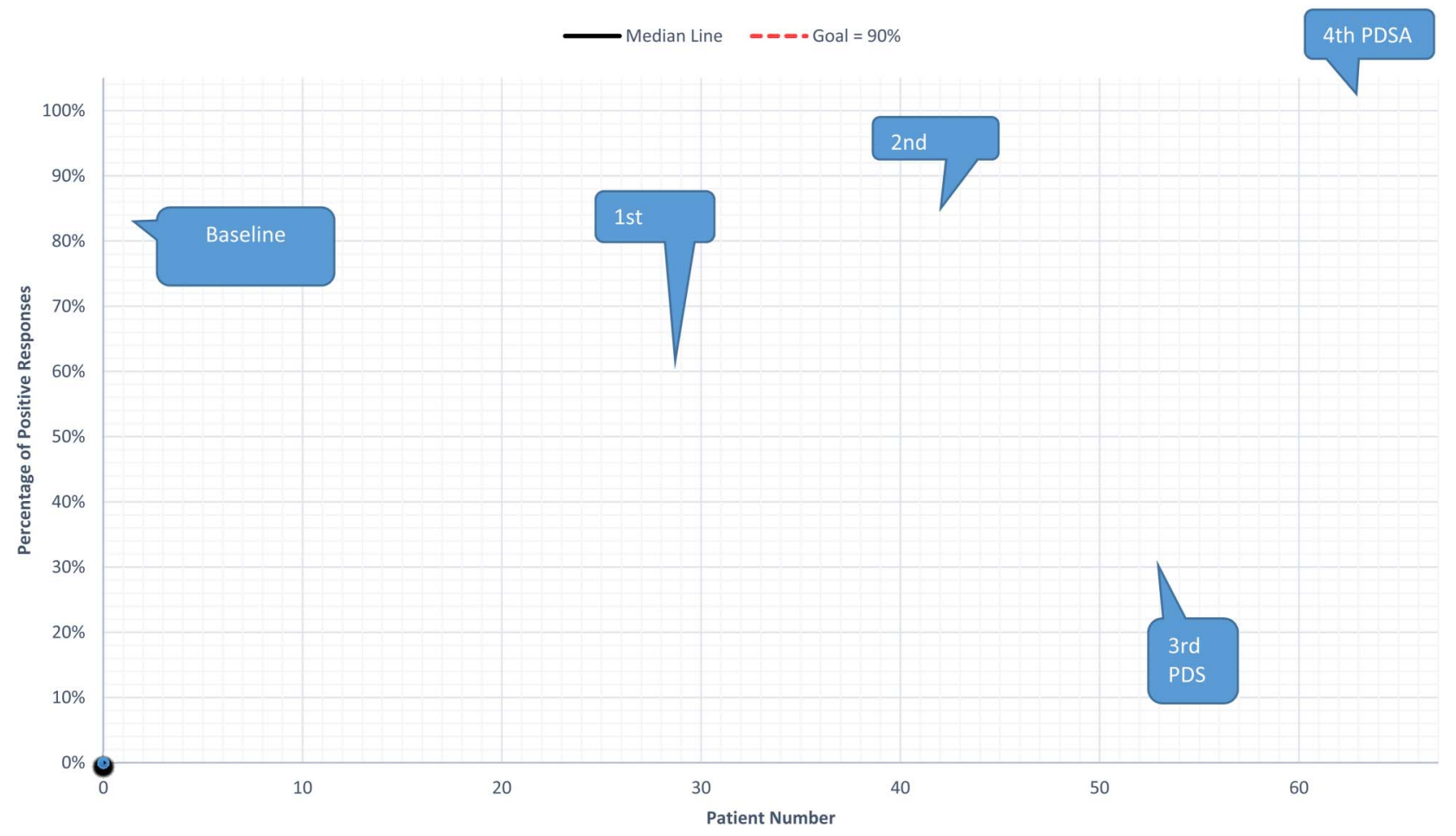


Despite all these improvements, actually ensuring the patient reads and understands the leaflet is difficult. Many thought that the leaflet was a quiz to be returned to the staff and others didn't understand the wording of some questions. Sentences must be simply worded with no medical jargon, and instructions to patients should be explicit and clear. This is particularly important for those patients in pain or the elderly.

A key factor in the success of the question paper was the timing of distribution to the patient. If given too early in the hospital stay, the patient is often too unwell to read it or take any notice, however if given on the day of discharge there is little opportunity for questions to be asked. Another limitation to the project was the difficulty of making appropriate contact to patients by phone. Many people did not answer their phone or the wrong number was recorded on the hospital database. This decreased our sample size.

A key limitation to the project was the declining number of leaflets given out to patients as the PDSA cycles progressed. When carried out by the study authors, the leaflets were given to as many patients as possible since a higher the number of patients would make a more representative study. When the distribution was outsourced to the ward doctors, ward clerk or ward manager, the number of leaflets successfully given out was much lower. It became apparent that implementing change to people's daily routines is difficult especially when the change is not a requirement.

The final PDSA cycle is encouraging as it shows that more leaflets were distributed by the nursing staff on the SAU. Increasing the distribution rate required profound encouragement through visits the ward and to remind staff about the leaflet. The distribution rate of $47 \%$ over 60 hours was much higher in this PDSA cycle compared to earlier cycles when only a handful of leaflets were distributed over a couple of weeks.

Our results do show an improvement in patient understanding from our baseline measurement however this could be due to chance or random fluctuations.

The lessons we have learned will be used towards achieving our goal of implementing the project hospital wide. We aim to gradually widen the scope of our project starting with all surgical wards and extending to general medical, maternity and paediatric wards. We can foresee that generalising the project may be limited by differences in patient groups on other wards. For example, the extended length of stay for some patients on the medical wards may lead to the leaflet being misplaced and geriatric patients may have cognitive impairment preventing them from understanding the leaflet.

To ensure sustainability going forwards we will communicate with ward staff regarding the aims of the leaflet and the most appropriate method of distribution on the ward. We will discuss the project with hospital managers and clinical leads in order to increase awareness of the project and create interest with the aim of making the leaflet a routine part of inpatient care.

\section{CONCLUSION}

Through personal experience during foundation year training, it is apparent that a surprising number of patients do not know basic information about their hospital admission. This anecdotal evidence is well supported by formal studies into patient knowledge about their diagnoses and medication. Horwitz et al found that only $59.6 \%$ of patients could accurately record their diagnosis and future management, in our study we found that prior to intervention, $69 \%$ of patients knew their diagnosis and future management, but testing this for accuracy was not in our study's remit. ${ }^{14}$

Once a patient is educated about their medical condition, they may feel empowered to take control of their own health and self manage expected symptoms at home. Educating the patient enables informed choices to be made about whether they want to take prescribed medications in view of the side effects or other problems that they may face as a result.

Reducing readmission rates is a complex problem, with many contributary factors, including age, socioeconomic status and morbidity. Many suggestions have been made to improve these rates including hospital at home and personalised health care plans. ${ }^{8}$ These are expensive interventions which would cost the NHS an enormous amount of money. Our intervention is relatively inexpensive (costing the price of one A4 sheet of paper and the time taken hand it to the patient) that could do well to improve one of the NHS' key aims of improving the patient experience as well as saving money in a cash-strapped system.

The final aim of the project is for the distribution of our question leaflet to become a part of daily routine in Derriford hospital. We aim to build on the lessons learned and information gained from the previous PDSA cycles in order to do this. A hospital-wide leaflet is a step towards improved local patient education and a more satisfied patient group.

Acknowledgements We would like to thank the nursing staff on Wolf and Stonehouse ward at Derriford hospital for their assistance and co-operation with our project.

\section{Declaration of interests Nothing to declare}

Ethical approval The NHS Health Research Authority uses the following criteria for determining if activities require ethics review (http://www. hra-decisiontools.org.uk/research/):

Policy criterion: Are the patients randomised? Is the patient care or treatment changed from accepted standards? Are the results are generalisable to broader patient populations?

Explanation: The work reported here is not classified as research and therefore does not require ethical approval as all patients were treated equally and not randomised, there was no change to their medical care and the results of this quality improvement project are only of interest in this specific clinical area. Further projects would have to be done to see if this is practical elsewhere, so it is not generalisable to broader populations.

Open Access This is an open-access article distributed under the terms of the Creative Commons Attribution Non-commercial License, which permits use, distribution, and reproduction in any medium, provided the original work is properly cited, the use is non commercial and is otherwise in compliance with the license. See: 
- http://creativecommons.org/licenses/by-nc/2.0/

- http://creativecommons.org/licenses/by-nc/2.0/legalcode

\section{REFERENCES}

1. Younis J, Salerno G, Chaudhary A, Trickett JP, Bearn PE, Scott HJ, Galbraith KA. Reduction in Hospital Reattendance due to Improved Preoperative Patient Education Following Hemorrhoidectomy. Journal For Healthcare Quality 2013;35:24-9.

2. Meredith C, Symonds P, Webster L, Lamont D, Elspeth P, Gillis CR, Fallowfield L. Information needs of cancer patients in west Scotland: cross sectional survey of patients' views. BMJ 1996;313:724.

3. Calkins DR, Davis RB, Reiley P, Phillips RS, Pineo KLC, Delbanco TL, Lezzoni LI. Patient-Physician Communication at Hospital Discharge and Patients' Understanding of the Postdischarge Treatment Plan. Arch Intern Med 1997;157:1026-30.

4. Horwitz LI; Moriarty JP, Chen C, Fogerty RL, Brewster UC, Kanade S, Ziaeian B, Jenq GY, Krumholz HM. Quality of Discharge Practices and Patient Understanding at an Academic Medical Center. JAMA Internal Medicine 2013:173:1715-22.

5. Effing T, Monninkhof EEM, van der Valk PP, Zielhuis GGA, Walters $\mathrm{EH}$, van der Palen JJ, Zwerink M (2007). 'Self-management education for patients with chronic obstructive pulmonary disease (Cochrane Review)'. Cochrane Database of Systematic Reviews, article CD002990. DOI: 10.1002/14651858.CD002990. pub2

6. Boulding W, Glickman SW, Manary MP, Schulman KA, Staelin R Relationship between patient satisfaction with inpatient care and hospital readmission within 30 days. Am J Manag Care 2011;17: 41-8.

7. NHS Confederation. The impact of non-payment for acute readmissions. Issue number:211,2011.

8. Shepperd S, Doll H, Broad J, Gladman J, lliffe S, Langhorne P, Richards S, Martin F, Harris R (2009b). 'Early discharge hospital at home (Cochrane Review)'. Cochrane Database of Systematic Reviews, issue 1, article CD000356. DOI: 10.1002/14651858. CD000356.pub3 